\title{
Los sistemas alternativos y aumentativos de comunicación (SAAC) como instrumento para disminuir conductas desafiantes en el alumnado con TEA: estudio de un caso
}

\author{
Alternative and augmentative communication systems (AACS) as \\ an instrument for decreasing challenging behaviour to students with \\ ASD: a case study
}

\section{Resumen}

Se describe una intervención, basada en el Programa de Habla Signada de Benson Schaeffer, para dotar de un Sistema Alternativo y Aumentativo de Comunicación (SAAC) a una niña de ocho años diagnosticada con trastorno del espectro autista (TEA). Se pretende que la alumna, mediante la utilización de signos, pueda expresar sus necesidades y disminuir las conductas desafiantes que propicia, ya que se estima que estas tienen una clara intención comunicativa. Se utiliza una metodología cualitativa de estudio de caso único y por objetivos. El programa se desarrolló en el aula de comunicación y lenguaje (CyL) de un centro público de educación infantil y primaria (CEIP) de la Comunidad Valenciana. Se instruyeron ocho signos que utiliza de manera funcional en diferentes contextos. Además, se evidencia una reducción de las conductas desafiantes con la introducción de los mismos.

\section{Palabras clave}

Trastorno del espectro autista, comunicación, conductas desafiantes, sistema alternativo y aumentativo de comunicación.

\begin{abstract}
An intervention based on the Benson Schaeffer's Speak Signal Program, to provide an eight years old girl with Autism Spectrum Disorder (ASD) with the Alternative and Augmentative Communication System (AACS) is described. The intervention aims at promoting the use of signs to express her needs and through this achieve a decrease in challenging behaviors, mostly produced when trying to communicate. A qualitative single case study by objective methodology was used. The program was developed in the communication and language classroom of a public school for children in nursery and primary education in Valencia. Eight signs worked functionally in different contexts. Likewise, reduction of challenging behavior was evident.
\end{abstract}

Keywords

Autism spectrum disorder, communication, challenging behaviors, alternative and augmentative communication system.

\author{
Rosa Mira Pastor \\ <romipas@alumni.uv.es> \\ Universidad de Valencia

\section{Claudia Grau} \\ <claudia.grau@uv.es> \\ Universidad de Valencia
}

Para citar:

Mira, R. y Grau, C. (2017): “Los sistemas alternativos y aumentativos de comunicación (SAAC) como instrumento para disminuir conductas desafiantes en el alumnado con TEA: estudio de un caso". Revista Española de Discapacidad, 5 (I): II 3-I32.

Doi: <https://doi.org/IO. 5569/23405 I04.05.01.07>

Fecha de recepción: I3-09-20I6 Fecha de aceptación: I0-03-20I7 


\section{Introducción}

La comunicación es indispensable para los seres humanos, ya que nos permite iniciar interacciones, expresar necesidades y deseos, y compartir experiencias y emociones. Este carácter indispensable hace que, en ocasiones, se dé por hecho que todas las personas tienen esta habilidad para comunicarse. No obstante, en la práctica clínica se puede comprobar que el proceso de comunicación no es automático en todas las personas (Chiang, 2008; Garrido et al., 201 5).

En concreto, en el trastorno del espectro autista (TEA) se evidencia un déficit en el área del lenguaje y la comunicación social. Se trata de un trastorno neuropsicológico asociado frecuentemente a discapacidad intelectual, que se manifiesta antes de los tres años de edad y que presenta una alteración cualitativa de la interacción social y de la comunicación, patrones conductuales restringidos, repetitivos y estereotipados con diferentes niveles de gravedad que pueden manifestarse a través de movimientos o habla repetitiva, inflexibilidad en las rutinas o patrones de comportamiento regulares (López y Rivas, 20I4; Ganz et al., 20I 5 ).

En el área socioemocional, las personas con TEA realizan acercamientos sociales anormales y fracasan en el inicio o en la respuesta a interacciones sociales. También pueden tener alteradas las conductas comunicativas verbales y no verbales, por lo que el habla y el lenguaje pueden no aparecer o retrasarse a pesar de que existan capacidades intelectuales evidentes. Además, tienen dificultades para adaptar su comportamiento a diferentes contextos sociales y presentan un escaso interés por otras personas (APA, 20I3).

Existe una gran variabilidad en los niveles lingüísticos estructurales de los niños con TEA, desde aquellos que presentan un vocabulario relativamente extenso y rico (con ecolalia y variaciones prosódicas y fonológicas) hasta otros que muestran incapacidad total para el habla
(Chiang, 2008; Ganz et al., 20I 5). De hecho, una parte significativa de las personas con autismo (del 30 al $50 \%$ ) nunca llega a adquirir un nivel de lenguaje suficiente para cubrir sus necesidades comunicativas diarias (Moreno, 20I3).

Los problemas de comunicación relacionados con el TEA varían según el desarrollo intelectual y social de la persona y pueden explicarse por (Martos y Ayuda, 2002):

a. La tendencia a centrarse en los detalles (teoría de la coherencia central débil): procesan los estímulos de forma local, acentuando el interés por las partes o los detalles y por tanto, percibiendo la información de forma distinta al resto (Verpoorten et al., 20I4).

b. El déficit en la función ejecutiva: presentan una alteración grave en la planificación de comportamientos, que les impide utilizar el lenguaje de forma espontánea para autorregular sus acciones (Ibáñez, 2005; Calderón et al., 20I2).

c. Déficits en la capacidad de inferir, predecir y atribuir estados mentales a otras personas (teoría de la mente): lo que provoca la evitación del contacto social, relaciones inadecuadas por no entender lo que las otras personas piensan, sienten o quieren, y alteraciones en el discurso y lenguaje no verbal (Calderón et al., 20I 2; Villanueva et al., 2016).

d. Alteraciones en la atención conjunta: tienen problemas para coordinarse entre los interlocutores, no aprenden habilidades sociales (reírse, mirar a los ojos, emitir sonidos, etc.) en contextos de interacción antes de empezar a hablar, y no construyen esquemas comunicativos que son fundamentales para el posterior desarrollo del lenguaje (Escudero et al., 2013; Garrido et al., 20I 5 ; Jiménez, 20I 5 ).

Por tanto, aunque las personas con TEA desarrollen el lenguaje verbal, tienen dificultades para atribuir correctamente los significados y obtener una comunicación 
efectiva y funcional. Necesitan ayuda para comprender las tareas y transiciones, para anticipar el futuro, y hacer del lenguaje una herramienta para la comunicación espontánea y funcional (Chiang, 2008; Barrios, 2013).

Por este motivo, se hace indispensable el uso de una comunicación alternativa y/o aumentativa que contribuya a que las personas con autismo inicien intercambios de comunicación con mayor facilidad y de manera estructurada y predecible (Ganz et al., 20I 2; González et al., 20I4; Ganz et al., 20I 5). Asimismo, la organización visual y clara de las actividades y del entorno convierte el contexto en un lugar más comprensible que pueden manejar, disminuyendo su ansiedad y frustración (Hogdgon, 2002).

Hay que construir entornos de aprendizaje estructurados en los que el alumnado pueda desarrollar habilidades de comunicación funcional (Fortea et al., 20I 5). Así, la metodología TEACCH (Treatment and Education of Autistic related Communication Handicapped Children) favorece la organización del tiempo, del espacio y las secuencias de acontecimientos en el entorno (Mesibov y Howley, 20I I). Pretende enseñar los acontecimientos de forma predecible y desarrollar actividades útiles para la vida, buscando el mayor grado de independencia posible en el contexto escolar y familiar (Ferrer et al., 2010).

\section{- Conductas desafiantes}

La enorme heterogeneidad sintomática que presentan las personas con TEA en el lenguaje y la comunicación da lugar a un espectro de funcionamiento amplio: desde la ausencia de conductas con intencionalidad comunicativa hasta la utilización de conductas de mayor complejidad funcional y formal que, en la mayoría de casos, constituyen las conductas desafiantes o inadaptadas (Martos y Ayuda, 2002).

Las conductas desafiantes o problemáticas son aquellas que, por su intensidad, duración o frecuencia, afectan negativamente al desarrollo personal del individuo y a las oportunidades de participación en la comunidad (Márquez, 2000; López et al., 20I4). Son comportamientos y hábitos aprendidos que se utilizan para compensar las escasas estrategias de las que disponen los niños con TEA (Muñoz, 20I3). En la mayoría de casos, la conducta desafiante es una forma de comunicar, de solicitar un resultado y de expresar las dificultades, necesidades, miedos o apetencias (Magiati y Howlin, 2003; Chiang, 2008; Matson et al., 2009, 20I3). Por tanto, es la consecuencia de su dificultad para comunicar sus frustraciones o ansiedades y establecer relaciones sociales de forma adecuada.

En las personas que no disponen de lenguaje verbal, estas conductas pueden acentuarse (Etchepareborda, 200I). Su única vía de comunicación será presentar conductas inadaptadas como gritos, golpes o autolesiones. Así, a menor nivel comunicativo, mayor presencia de conductas disruptivas (Emerson y Einfeld, 20I I; O’ Reilly et al., 20I 2; Matson et al., 2013).

En consecuencia, una de las claves fundamentales para la prevención de las conductas problemáticas es la intervención comunicativa (Carr et al., I996; Magiati y Howlin, 2003). En concreto, la comunicación aumentativa y/o alternativa y la organización del entorno y de las actividades refuerzan el lenguaje de los niños, reducen los efectos que obstaculizan la comprensión, disminuyen la frustración ante el aprendizaje y favorecen el uso funcional del lenguaje (O'Reilly et al., 20I2; Banoy, 20I3; Ganz et al., 20I 5). Por tanto, la enseñanza de un lenguaje alternativo al oral permite que el alumno se comunique de forma funcional y que mejore su comportamiento, disminuyendo las conductas inadaptadas.

\section{- Sistemas alternativos y/o aumentativos de comunicación}

Los SAAC tienen como objetivo enseñar, mediante procedimientos específicos de instrucción, un conjunto estructurado de 
códigos no vocales que pueden necesitar o no soporte físico y permiten llevar a cabo actos de comunicación funcional, espontánea y generalizable (Ganz et al., 20I 2, 20I 5 ; González et al., 20I4). Son métodos destinados a potenciar cualquier habilidad comunicativa (Martos y Llorente, 20I3).

En la elección del SAAC se debe tener en cuenta el perfil cognitivo, perceptivo y social del alumno y la naturaleza de los mecanismos de compensación, espontáneos o inductivos (Monfort, 2009). Por tanto, antes de introducir un SAAC debe existir una evaluación de sus capacidades, habilidades y necesidades, y de las demandas de su entorno familiar y escolar. Hay que seleccionar de forma individualizada las estrategias de intervención y el vocabulario signado o pictográfico (Valencia, 20I4).

En las personas con TEA, como tienen dificultades para procesar la información auditiva que cambia rápidamente, hay que utilizar sistemas aumentativos que combinen la palabra con apoyos visuales. De este modo, disponen de mensajes no transitorios que persisten durante el tiempo que se tarda en procesar la información y se favorece el desarrollo del lenguaje oral (Fortea et al., 2015; Ganz et al., 20I 5 ).

Así pues, la aplicación de este tipo de sistemas proporciona a las personas no hablantes un medio eficaz de comunicación para que puedan convertirse en comunicadores activos. Este hecho dará lugar a una mejor calidad de vida, al desarrollo de su autonomía y favorecerá sus relaciones interpersonales y su autoestima (Regis y Callejón, 2015).

En el caso del alumnado con TEA, el sistema de Comunicación Total es uno de los más utilizados y con mejores resultados según evidencias basadas en la práctica (García y Sánchez, 2003; Monfort, 2006; Banoy, 20I3; Fortea et al., 20I5; Regis y Callejón, 20I5).

Este programa enfatiza la espontaneidad y el lenguaje expresivo y se estructura a través del aprendizaje de funciones lingüísticas: expresión de los deseos, referencia, conceptos de persona, petición de información, abstracción, juego simbólico y conversación (Schaeffer et al., 2005). Incluye dos componentes: el habla signada (producción por parte del niño de habla y de signos de forma simultánea) y la comunicación simultánea (utilización, por parte del adulto, de dos códigos simultáneamente: código oral y código signado) (Regis y Callejón, 20I5).

Está diseñado para fomentar tres áreas del desarrollo del lenguaje: aprendizaje del lenguaje signado, la potenciación del uso del habla signada y el desarrollo del lenguaje verbal espontáneo. Es por ello que su aprendizaje y utilización no entorpece, frena o dificulta la aparición del lenguaje verbal, sino que favorece e influye en el desarrollo del mismo (Fortea et al., 201 5). En la primera fase (lenguaje de signos) se enseña a utilizar el signo para pedir un determinado objeto. En primer lugar, el instructor moldea completamente el signo (con los tres componentes) y, poco a poco, va retirándole las ayudas táctiles siguiendo el encadenamiento hacia atrás hasta que lo realiza de forma totalmente autónoma. Asimismo, el instructor moldea el signo al receptor acompañándolo de su verbalización y de los movimientos del signo correspondientes a cada una de las sílabas que lo componen (Schaeffer et al., 2005).

La enseñanza de signos se lleva a cabo mediante el moldeamiento y la técnica del encadenamiento hacia atrás (Regis y Callejón, 201 5), que permiten la adquisición de cada signo y su uso espontáneo (Schaeffer et al., 2005). Se potencia el aprendizaje sin error, ya que se favorecen las intenciones comunicativas correctas mediante la consecución de aquello a lo que hace referencia el signo y se adaptan los objetivos al nivel evolutivo de las personas con TEA, para que estos sean asimilados correctamente y sin errores (Soto, 2007). Se planifican actividades llamativas que mantengan la motivación y se utilizan reforzadores potentes que sean significativos (Valencia, 20I4).

Ahora bien, ¿Hasta qué punto es eficaz el programa de Comunicación Total de Schaeffer? 
¿Las conductas problemáticas tienen realmente una función comunicativa? ¿Pueden verse reducidas el número de conductas desafiantes con la introducción de los SAAC? Estos interrogantes son los que nos planteamos y queremos responder en nuestra investigación mediante el programa de intervención.

\section{Método}

En la presente investigación se ha diseñado un programa de intervención para dotar a una niña con TEA, de ocho años, de un SAAC y comprobar si con la dotación de este se reducen sus conductas desafiantes. En concreto, se ha implementado la primera fase del programa de Habla Signada de Schaeffer. Se ha utilizado una metodología de estudio de caso único la cual implica un examen detallado y sistemático del mismo.

\subsection{Hipótesis y objetivos}

Este trabajo sustenta su intervención en la siguiente hipótesis: la introducción de los SAAC ayuda a cubrir el déficit comunicativo que presentan las personas con TEA y, como consecuencia, se reducen las conductas desafiantes.

\section{Objetivos}

- Evidenciar la presencia de conductas desafiantes con intención comunicativa.

- Diseñar, poner en práctica y evaluar un programa de intervención basado en el programa de Habla Signada para alumnos no verbales de Benson Schaeffer, con el objeto de favorecer la comunicación funcional de una alumna no verbal y disminuir las conductas desafiantes.

\subsection{Contexto}

La investigación se ha desarrollado en un aula de Comunicación y Lenguaje $(\mathrm{CyL})$ situada en un CEIP público de la ciudad de Valencia. Está conformada por cinco alumnos que pasan el $90 \%$ de las horas lectivas en ésta (modalidad A) y otros tres, un porcentaje menor al $40 \%$ (modalidad B), complementando su escolarización en el aula ordinaria de referencia. De entre los cinco alumnos de modalidad A, únicamente uno de ellos ha desarrollado el lenguaje verbal, mientras que el resto no dispone de un medio eficaz para comunicarse.

El aula se encuentra adaptada a sus necesidades, ya que la enseñanza se basa en la metodología TEACCH. Para llevar a cabo las diferentes sesiones de intervención, se ha habilitado una zona específica del aula CyL denominada "rincón de comunicación".

\subsection{Participantes}

De entre los alumnos que acuden al aula CyL se ha seleccionado a una niña de ocho años diagnosticada con TEA (a la que llamaremos S). Actualmente se encuentra escolarizada en un CEIP público y acude al aula CyL en la modalidad A.

\section{El diagnóstico (realizado en 20I I) es} Trastorno Generalizado del Desarrollo $\left(\mathrm{TGD}^{\mathrm{I}}\right.$ ) no especificado, junto con retraso mental moderado. Los instrumentos utilizados para su evaluación psicopedagógica fueron los criterios del DSM-IV-TR, a través de la observación directa de la niña en el contexto escolar durante el primer curso de educación infantil. Además, se utilizaron los cuestionarios: Escala de desarrollo Brunet-Lezine y Escala de Evaluación del Autismo GARS-2 (20I I), coincidiendo en que el área de comunicación y lenguaje es la más afectada.

\footnotetext{
I. Según establece el manual diagnóstico de referencia (DSM5), “a los pacientes con un diagnóstico bien establecido según el DSM-IV de trastorno autista, enfermedad de Asperger o TGD no especificado, se les aplicará el Trastorno del Espectro Autista como diagnóstico" (APA, 2013: I00).
} 
Por lo que respecta a la historia escolar, los dos primeros cursos de educación infantil los cursó en educación combinada. A partir del tercer curso se escolarizó en el aula CyL del CEIP donde actualmente cursa segundo de educación primaria.

Sin finalidades diagnósticas, se pueden exponer las características que presenta $S$ según los criterios diagnósticos del manual DSM-5 (APA, 20I3) en referencia al área de comunicación y lenguaje. Presenta déficits persistentes en la comunicación por la ausencia del lenguaje verbal que, en la mayoría de casos, desemboca en conductas inadaptadas, ya que éstas constituyen la forma de expresar sus necesidades. En el campo de la interacción social, la alumna presenta carencias en la forma de socializar, ya que la mayoría de intentos de interacción social son llevados a cabo mediante gritos. No obstante, estos gritos continuados también indican que hay intención de interactuar con el resto.

Las únicas demandas orales que realiza de forma espontánea son “aah”, utilizada para pedir alguna cosa, reclamar atención o cuando se enfada; y "uh" o "ba" para señalar algo determinado. A pesar de ello, $S$ muestra un grado bastante alto de intención comunicativa que se evidencia en la utilización de las tres únicas demandas orales de las que dispone de forma persistente. En referencia al desarrollo social, la niña muestra abundantes acercamientos con sus iguales e interés por los mismos. A pesar de ello, no utiliza el lenguaje oral en ningún caso, ya que se limita al uso de algunos gestos (ven, adiós, hola y señalar lo que quiere) aprendidos por la buena capacidad de imitar a la gente de su entorno.

En referencia al lenguaje receptivo, necesita claves visuales que le faciliten la comprensión de las tareas a realizar. No obstante, es capaz de asociar enunciados verbales a órdenes o peticiones por parte del adulto. Sin embargo, en ocasiones ignora el lenguaje y no hay respuesta a órdenes o indicaciones, y otras veces se niega a realizar la instrucción con un conjunto de conductas desafiadoras como gritos descontrolados, autolesiones o golpes. Por este motivo, la posterior intervención se inicia con una evaluación funcional de las conductas problemáticas de la alumna, las cuales denotan un alto porcentaje de intención comunicativa. Este hecho no hace más que evidenciar la necesidad de intervenir en este campo para reducir los déficits comunicativos y para que, como consecuencia, se reduzcan las conductas inadaptadas que presenta.

\subsection{Instrumentos}

En la investigación se han utilizado:

- Las entrevistas informales para recabar información. Se hicieron preguntas abiertas a las profesionales del aula CyL (maestra de pedagogía terapéutica y de audición y lenguaje, y educadora) sobre los problemas y déficits que presentaba $S$, así como sus gustos y preferencias. Esto permitió definir las conductas a observar: autolesión, agresión, perturbación (gritar y destruir) e incumplimiento (levantarse).

- Observación. Se ha realizado mediante una tabla de registro en la que se anota cada conducta problemática a lo largo del día durante el horario escolar. Se pretende recoger el tipo (autolesión, agresión, perturbación -gritar y destruir- e incumplimiento -levantarse), la frecuencia y las funciones (obtener, evitar o llamar la atención) de las conductas, así como los elementos del contexto que las propiciaban (comunicación, frustración, aburrimiento y daño físico). Este registro se ha hecho durante dos semanas antes y después del programa de intervención.

- El vocabulario de signos. Se ha seleccionado en base a una evaluación formal de reforzadores, a través de: a) entrevista informal a las personas que constituyen el entorno de la alumna para saber cuáles son sus intereses y preferencias, b) la observación de la alumna en situaciones o contextos naturales (tiempo de juego y de descanso) para definir los objetos y 
actividades que le gustan, y c) evaluación formal de reforzadores: se le han mostrado objetos (seleccionados a partir de la información recogida) y se ha anotado su reacción ante ellos (rechaza, ninguna reacción, intenta cogerlo, muestra señales de que le gusta, lo vuelve a coger) para seleccionar los primeros signos a instruir.

- Observación en distintos contextos del centro escolar. Se ha utilizado una tabla de adquisición de signos para valorar el grado de consecución de cada signo instruido (Tabla I).

Tabla 1. Ítems para evaluar la adquisición de los signos

\begin{tabular}{|l|l|}
\cline { 2 - 2 } \multicolumn{1}{c|}{} & $\begin{array}{l}\text { Criterios para evaluar la } \\
\text { adquisición de signos }\end{array}$ \\
\hline Discriminación & $\begin{array}{l}\text { Utiliza el signo que hace } \\
\text { referencia al objeto/acción que } \\
\text { quiere pedir y no lo confunde con } \\
\text { otros. }\end{array}$ \\
\hline Uso funcional & $\begin{array}{l}\text { Utiliza el signo para pedir aquello } \\
\text { que desea, incluso cuando el } \\
\text { referente no está delante. }\end{array}$ \\
\hline $\begin{array}{l}\text { Generalización } \\
\text { a otros } \\
\text { contextos }\end{array}$ & $\begin{array}{l}\text { Utiliza el signo en otros entornos } \\
\text { del centro escolar (patio, tiempo } \\
\text { de juego...) y no solo en la zona } \\
\text { de entrenamiento del aula CyL. }\end{array}$ \\
\hline $\begin{array}{l}\text { Generalización } \\
\text { a otras } \\
\text { personas }\end{array}$ & $\begin{array}{l}\text { Utiliza el signo para pedir aquello } \\
\text { que desea a cualquier persona } \\
\text { (compañeros, maestras...) y no } \\
\text { solo al entrenador. }\end{array}$ \\
\hline
\end{tabular}

Fuente: elaboración propia.

\subsection{Procedimiento}

En primer lugar se ha cumplimentado la tabla de registro de conductas, previa a la intervención. Teniendo en cuenta que el déficit más representativo es el comunicativo, se ha decidido introducir un SAAC. En la selección del SAAC se ha valorado la capacidad de imitación de la alumna, la intencionalidad comunicativa de algunas de sus conductas inadaptadas y la ausencia de dificultades motrices. Así, se ha diseñado y puesto en práctica un plan de intervención basado en el programa de Habla Signada para alumnos no verbales (Schaeffer et al., 2005). Se le ha enseñado a utilizar determinados signos para que $S$ pueda pedir aquello que desee sin tener que recurrir a conductas inadaptadas y se han seleccionado los signos en base a una jerarquía de reforzadores (vocabulario de signos).

Finalmente, para comprobar si con la dotación del SAAC han disminuido las conductas inadaptadas, se ha vuelto a cumplimentar la tabla de registro observacional de las conductas después de la intervención.

\section{- Descripción del programa de intervención}

Se trabaja únicamente la primera fase del programa de Habla Signada (entrenamiento del lenguaje signado). Se seleccionan los signos que son más motivadores, para que consiga realizar las peticiones de forma satisfactoria. Los signos elegidos han sido: caramelo, beber, pelota, gusanito, música, pompas, galleta y crema.

La intervención se ha llevado a cabo durante tres semanas en el horario escolar. Cada sesión está programada para enseñar un signo diferente y su duración se adapta al ritmo en que se van adquiriendo los signos. Asimismo, se intercalan sesiones de repaso, consistentes en la repetición de la sesión anterior y en la creación de entornos que propicien la comunicación (juegos poco estructurados u objetos fuera del alcance) y comprobar si utiliza el signo de forma funcional (Tabla 2). 


\begin{tabular}{|c|c|c|c|c|}
\hline \multicolumn{5}{|l|}{ Abril 2016} \\
\hline $\mathbf{L}$ & M & M & $J$ & v \\
\hline & & & & 1 \\
\hline 4 & $\begin{array}{l}5 \\
\text { Sesión 1: Caramelo }\end{array}$ & $\begin{array}{l}6 \\
\text { Repaso de caramelo }\end{array}$ & $\begin{array}{l}7 \\
\text { Sesión 2: Beber }\end{array}$ & $\begin{array}{l}8 \\
\text { Sesión 3: } \\
\text { Discriminación de } \\
\text { signos }\end{array}$ \\
\hline $\begin{array}{l}11 \\
\text { Sesión 4: Pelota }\end{array}$ & $\begin{array}{l}12 \\
\text { Repaso de pelota }\end{array}$ & $\begin{array}{l}13 \\
\text { Sesión 5: Música }\end{array}$ & $\begin{array}{l}14 \\
\text { Sesión 6: } \\
\text { Discriminación de } \\
\text { signos }\end{array}$ & $\begin{array}{l}15 \\
\text { Sesión 7: Gusanito }\end{array}$ \\
\hline $\begin{array}{l}18 \\
\text { Sesión 8: Pompas }\end{array}$ & $\begin{array}{l}19 \\
\text { Repaso de pompas } \\
\text { y gusanito }\end{array}$ & $\begin{array}{l}20 \\
\text { Sesión 9: Galleta }\end{array}$ & $\begin{array}{l}21 \\
\text { Sesión 10: } \\
\text { Discriminación de } \\
\text { signos }\end{array}$ & $\begin{array}{l}22 \\
\text { Sesión 11: } \\
\text { Crema }\end{array}$ \\
\hline $\begin{array}{l}25 \\
\text { Repaso de crema }\end{array}$ & $\begin{array}{l}26 \\
\text { Sesión 12: Quiero } \\
\text { +objeto }\end{array}$ & 27 & 28 & 29 \\
\hline
\end{tabular}

Fuente: elaboración propia.

En total se han realizado doce sesiones de entrenamiento en las que se han instruido ocho signos como peticiones simples y en la última sesión una petición de dos componentes: "quiero + objeto/actividad". Asimismo, se han utilizado ayudas táctiles (durante el moldeamiento), verbales (al pronunciar la palabra mientras la alumna realiza el signo), visuales (con el objeto real o con pictogramas) e imitativas, y el propio objeto como reforzador.

\section{Sesión 1. El primer signo: una petición expresando un deseo}

El primer signo permite a la alumna expresar un deseo mediante una petición, por lo que tendrá que representar un alimento, actividad o juguete que desee intensamente, lo que incitará a producir el signo para conseguir aquello que desea (Ver Tabla 3).

\section{Sesión 2. El segundo signo: petición y descripción}

Se pretende que S aprenda a signarlo, a discriminarlo del primero y a utilizarlo para hacer una petición. Para que la alumna pueda discriminarlos, las palabras y los signos (en sus tres componentes) deben ser diferentes (Ver Tabla 4).

\section{Sesión 3. Primera sesión de discriminación}

Una vez la alumna ha adquirido de forma funcional los dos primeros signos, y después de comprobarlo en diferentes contextos, se introduce una sesión específica de discriminación de los dos signos (Ver Tabla 5). 


\begin{tabular}{|c|c|c|c|c|c|}
\hline \multicolumn{6}{|c|}{ Sesión 1: primer signo "caramelo" } \\
\hline \multirow{2}{*}{\multicolumn{2}{|c|}{$\begin{array}{l}\text { CARAMELO Girar el dedo indice } \\
\text { en el centrode la cara. }\end{array}$}} & \multicolumn{4}{|c|}{ Componentes } \\
\hline & & \multicolumn{4}{|c|}{$\begin{array}{l}\text { Posición: la mano en la mejilla. } \\
\text { Forma de la mano: con el puño cerrado y el dedo índice extendido. } \\
\text { Movimiento: rotar el dedo índice. }\end{array}$} \\
\hline \multirow{4}{*}{ Descripción } & \multicolumn{5}{|c|}{ Parte 1: ensayos en bloque } \\
\hline & \multicolumn{5}{|c|}{$\begin{array}{l}\text { Se le ofrece un caramelo y antes de que intente alcanzarlo, se le moldea el signo. Se realizan sucesivos entrenamientos, } \\
\text { retirándole poco a poco la ayuda y siguiendo el encadenamiento hacia atrás. }\end{array}$} \\
\hline & \multicolumn{5}{|c|}{ Parte 2: busco el objeto } \\
\hline & \multicolumn{5}{|c|}{$\begin{array}{l}\text { Después de unos ensayos de moldeamiento del signo, se colocan tres botes con pictogramas referidos a lo que } \\
\text { tienen en el interior (solamente uno contiene lo que designa el pictograma, en este caso, caramelos). Se ponen los } \\
\text { tres botes delante de la alumna y se le pregunta: ¿qué quieres? Ella elige uno de los botes, lo abre y ve que está } \\
\text { vacío, entonces escoge otro. Cuando escoge el de caramelos, este se le retira y se le pregunta: ¿qué quieres?, } \\
\text { ¿quieres caramelo? Repetimos el ejercicio varias veces hasta que lo pida de la forma correcta y sin ayudas. }\end{array}$} \\
\hline Errores & \multicolumn{5}{|c|}{$\begin{array}{l}\text { - En la posición: produce el signo en la frente y en la barbilla y no en la mejilla. } \\
\text { - En la forma: produce el signo con las dos manos, a pesar de que se le bloquee una. }\end{array}$} \\
\hline \multirow{2}{*}{ Duración } & \multirow{2}{*}{\multicolumn{2}{|c|}{20 minutos }} & \multirow{2}{*}{ Ensayos } & $\mathrm{N}^{\circ}$ & 20 \\
\hline & & & & Tipo & En bloque \\
\hline
\end{tabular}

Fuente: elaboración propia.

\begin{tabular}{|c|c|c|c|c|}
\hline \multicolumn{5}{|c|}{ Sesión 2: segundo signo "beber" } \\
\hline \multirow[b]{2}{*}{ 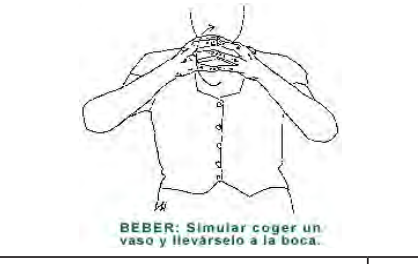 } & \multicolumn{4}{|c|}{ Componentes } \\
\hline & \multicolumn{4}{|c|}{$\begin{array}{l}\text { Posición: la mano enfrente de la boca. } \\
\text { Forma de la mano: las dos manos juntas con los dedos abiertos y las yemas de ambas manos tocándose. } \\
\text { Movimiento final: mover los brazos hacia arriba y hacia abajo. }\end{array}$} \\
\hline \multirow{6}{*}{ Descripción } & \multicolumn{4}{|c|}{ Parte 1: recordatorio de caramelo } \\
\hline & \multicolumn{4}{|c|}{$\begin{array}{l}\text { Se le ofrece un caramelo varias veces para ver si se acuerda del signo hasta que lo realice correctamente de } \\
\text { forma espontánea. }\end{array}$} \\
\hline & \multicolumn{4}{|c|}{ Parte 2: ensayos en bloque } \\
\hline & \multicolumn{4}{|c|}{$\begin{array}{l}\text { Se le ofrece un vaso con agua y antes de que la alumna intente alcanzarlo, la maestra le moldea el signo. Se } \\
\text { realizan entrenamientos sucesivos, retirándole poco a poco la ayuda. }\end{array}$} \\
\hline & \multicolumn{4}{|c|}{ Parte 3: generalizar el signo } \\
\hline & \multicolumn{4}{|c|}{$\begin{array}{l}\text { Se le enseñan tres vasos con distintas bebidas: agua, zumo y leche con su pictograma correspondiente. Se le } \\
\text { pide que haga el gesto para que entienda que todo se puede "beber". Se ponen los tres vasos delante de ella } \\
\text { y se le pregunta: ¿qué quieres? Se espera hasta que realice el signo "beber". }\end{array}$} \\
\hline Errores & \multicolumn{4}{|c|}{$\begin{array}{l}\text { - Errores de posición en el signo "caramelo". Realiza el signo debajo de los ojos y no en la mejilla, pero ya no } \\
\text { en la barbilla y en la frente (ha mejorado la posición). Aunque la forma de la mano es correcta, utiliza las dos } \\
\text { manos para designar el signo. } \\
\text { - Errores de discriminación entre el primer y segundo signo. Utiliza el signo "caramelo" en repetidas } \\
\text { ocasiones, en los ensayos del de "beber". }\end{array}$} \\
\hline \multirow{2}{*}{ Duración } & \multirow{2}{*}{$\begin{array}{l}25 \\
\text { minutos }\end{array}$} & \multirow{2}{*}{ Ensayos } & $\mathrm{N}^{\circ}$ & 15 \\
\hline & & & Tipo & En bloque \\
\hline
\end{tabular}

Fuente: elaboración propia. 
Tabla 5. Primera sesión de discriminación

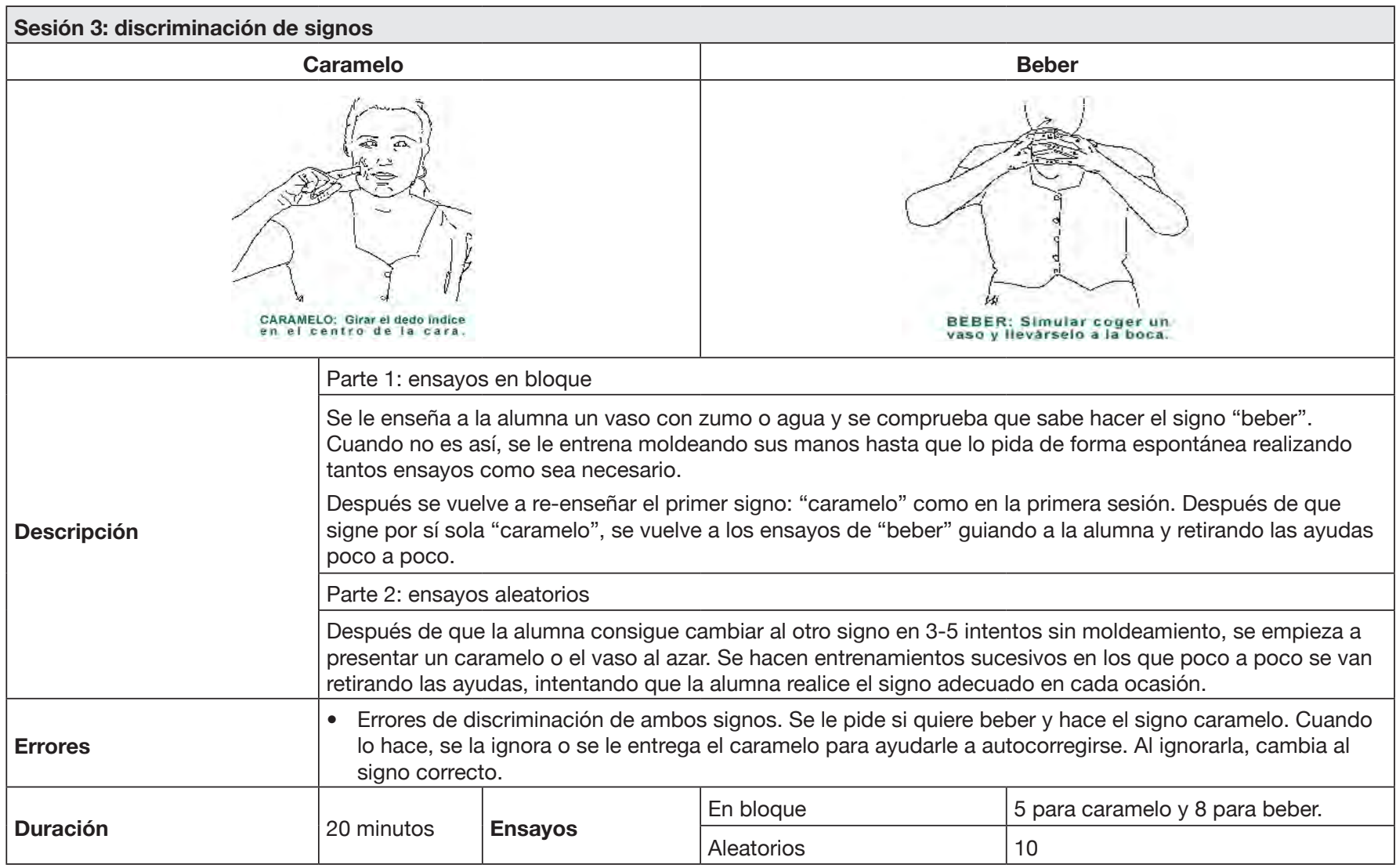

Fuente: elaboración propia.

\section{Sesiones de la 4 a la 11: tercer signo y} posteriores

Una vez adquiridos los dos primeros signos se van introduciendo los restantes de la misma forma (Ver Tabla 6). 


\begin{tabular}{|c|c|c|c|c|}
\hline \multicolumn{5}{|c|}{ Sesión 4: tercer signo "pelota" } \\
\hline \multirow{2}{*}{\multicolumn{2}{|c|}{$\begin{array}{l}\text { PELOTA: Mano abierta con la } \\
\text { palma hacia abajo baja y sube. }\end{array}$}} & \multicolumn{3}{|l|}{ Componentes } \\
\hline & & \multicolumn{3}{|c|}{$\begin{array}{l}\text { Posición: brazo estirado } \\
\text { Forma de la mano: la mano abierta con la palma hacia abajo. } \\
\text { Movimiento final: la mano sube y baja. }\end{array}$} \\
\hline \multirow{5}{*}{ Descripción } & \multicolumn{4}{|c|}{ Parte 1: ensayos en bloque } \\
\hline & \multirow{2}{*}{\multicolumn{4}{|c|}{$\begin{array}{l}\text { Se presenta una caja con } 3 / 4 \text { pelotas, de diferentes tamaños, colores y dibujos. Se le enseña una } \\
\text { pelota y, cuando intenta cogerla, se le moldea el signo. Tras sucesivos entrenamientos, se van } \\
\text { retirando poco a poco las ayudas, ofreciéndole el objeto y entregándoselo cuando haga el signo } \\
\text { correctamente hasta que signe espontáneamente. } \\
\text { A continuación, se le deja elegir una de las pelotas sin que llegue a cogerla. Se le pregunta } \\
\text { ¿quieres pelota? y se espera a que haga el gesto. Posteriormente, se deja la caja fuera de su } \\
\text { alcance para que la vuelva a pedir. }\end{array}$}} \\
\hline & & & & \\
\hline & \multicolumn{4}{|c|}{ Parte 2: rincón de juego } \\
\hline & \multicolumn{4}{|c|}{$\begin{array}{l}\text { Para propiciar la generalización del signo, nos desplazamos al rincón de juego y sentadas en una } \\
\text { colchoneta se le pregunta si quiere la pelota. Cuando intenta cogerla se le moldea el signo hasta } \\
\text { que lo utiliza de forma espontánea. Posteriormente, se le deja un momento para que juegue, lo que } \\
\text { contribuirá a la posterior petición de la pelota. }\end{array}$} \\
\hline Errores & \multicolumn{4}{|c|}{$\begin{array}{l}\text { - Errores en la forma del signo. No estira completamente el brazo, la mano la mantiene boca } \\
\text { abajo y realiza el movimiento adecuadamente, pero lo hace con las dos. }\end{array}$} \\
\hline \multirow{2}{*}{ Duración } & \multirow{2}{*}{20 minutos } & \multirow{2}{*}{ Ensayos } & $\mathrm{N}^{\circ}$ & 15 \\
\hline & & & Tipo & En bloque \\
\hline \multicolumn{5}{|c|}{ Sesión 5: cuarto signo "música" } \\
\hline & & \multicolumn{3}{|l|}{ Componentes } \\
\hline & & \multicolumn{3}{|c|}{$\begin{array}{l}\text { Posición: la mano situada a la altura de la oreja. } \\
\text { Forma de la mano: el puño cerrado con el dedo índice estirado. } \\
\text { Movimiento final: el dedo índice gira en el pabellón de la oreja. }\end{array}$} \\
\hline \multirow{4}{*}{ Descripción } & \multicolumn{4}{|c|}{ Parte 1: asociar música a radiocasete } \\
\hline & \multicolumn{4}{|c|}{$\begin{array}{l}\text { Cuando la alumna se sienta en la mesa, le quitamos el radiocasete y lo alejamos para que no } \\
\text { pueda alcanzarlo. En el momento en que intenta alcanzarlo, se le moldea el signo. De este modo, } \\
\text { propiciamos que nos pida el radiocasete mediante el signo "música". Poco a poco se le van } \\
\text { retirando las ayudas y ofreciéndole el radiocasete cuando lo haga de forma correcta hasta que } \\
\text { realice el signo de forma espontánea. }\end{array}$} \\
\hline & \multicolumn{4}{|c|}{ Parte 2: asociación del signo "música" a una canción } \\
\hline & \multicolumn{4}{|c|}{$\begin{array}{l}\text { Una vez ha realizado el signo de forma adecuada en cuanto a posición, forma y movimiento, se } \\
\text { introduce un cd en el radiocasete y se le pregunta: “¿quieres música?”. Se espera a que realice el } \\
\text { signo, prestándole las ayudas necesarias y posteriormente se pone la música. Se repite hasta que } \\
\text { la alumna realice el signo de forma autónoma. }\end{array}$} \\
\hline Errores & \multicolumn{4}{|c|}{$\begin{array}{l}\text { - Errores de posición: coloca la mano en la cabeza para designar "música". Sin embargo, la forma } \\
\text { de la mano la realiza de forma adecuada desde los primeros ensayos. } \\
\text { - Errores de discriminación: en algunas ocasiones confunde el signo "música" con el de } \\
\text { "caramelo" debido a la similitud de la forma de la mano de ambos signos. }\end{array}$} \\
\hline \multirow{2}{*}{ Duración } & \multirow{2}{*}{15 minutos } & \multirow{2}{*}{ Ensayos } & $\mathrm{N}^{\circ}$ & 10 \\
\hline & & & Tipo & En bloque \\
\hline
\end{tabular}




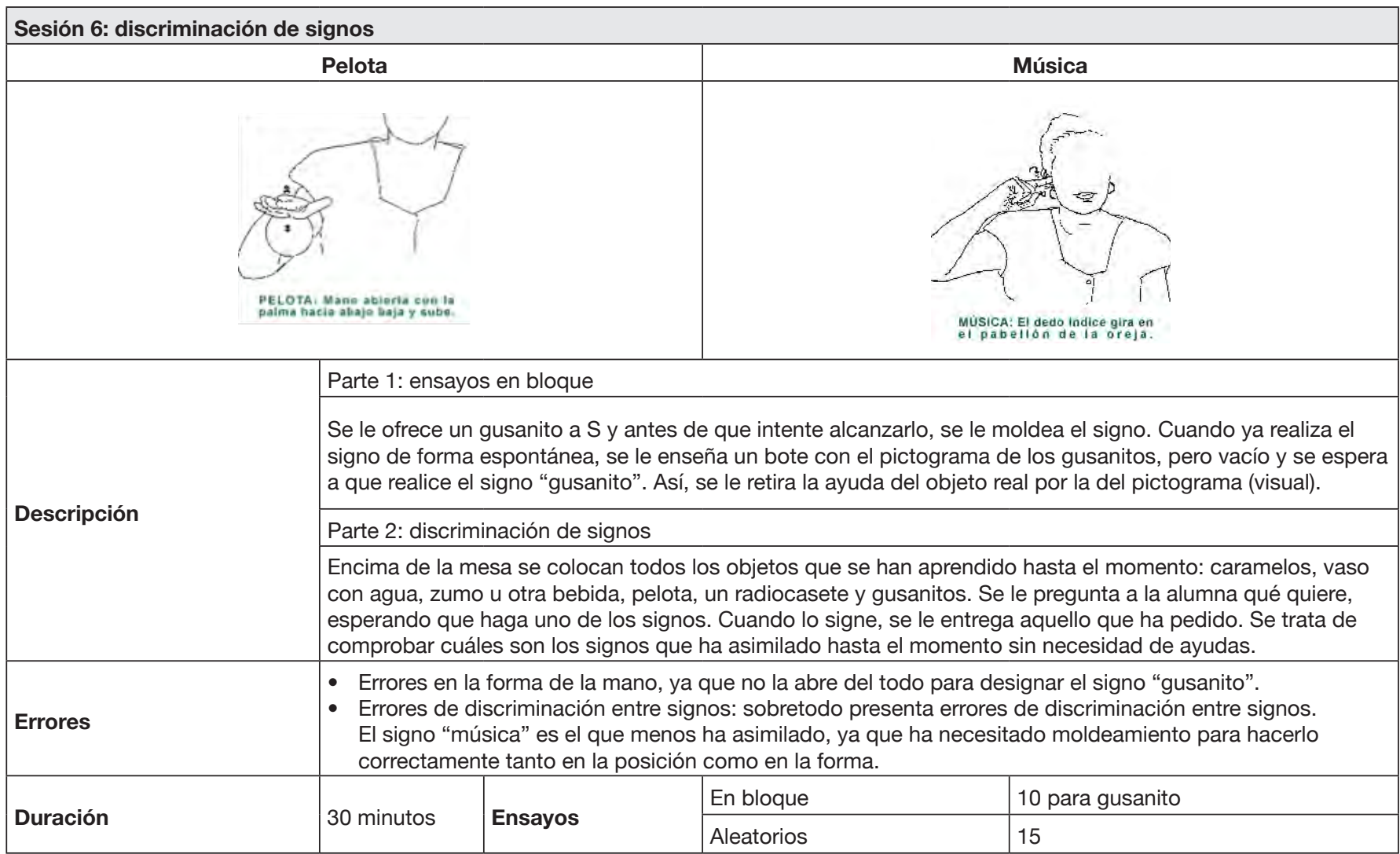

\begin{tabular}{|c|c|c|c|c|}
\hline \multicolumn{5}{|c|}{ Sesión 7: quinto signo "gusanito" } \\
\hline \multicolumn{3}{|c|}{$\begin{array}{l}\text { GUSANITOS: El dedo indice de una } \\
\text { mano golpea la palma de la otra. }\end{array}$} & \multicolumn{2}{|c|}{$\begin{array}{l}\text { 1. Posición: las dos manos a la altura del pecho. } \\
\text { 2. Forma de la mano: una mano abierta y la otra con el puño cerrado } \\
\text { y el dedo índice estirado. } \\
\text { 3. Movimiento final: el dedo índice de una mano golpea la palma de } \\
\text { la otra. }\end{array}$} \\
\hline \multirow{4}{*}{ Descripción } & \multicolumn{4}{|c|}{ Parte 1: ensayos en bloque } \\
\hline & \multicolumn{4}{|c|}{$\begin{array}{l}\text { Se le ofrece un gusanito a S y antes de que intente alcanzarlo, se le moldea el signo. Cuando ya realiza el } \\
\text { signo de forma espontánea, se le enseña un bote con el pictograma de los gusanitos, pero vacío y se espera } \\
\text { a que realice el signo "gusanito". Así, se le retira la ayuda del objeto real por la del pictograma (visual). }\end{array}$} \\
\hline & \multicolumn{4}{|c|}{ Parte 2: discriminación de signos } \\
\hline & \multicolumn{4}{|c|}{$\begin{array}{l}\text { Encima de la mesa se colocan todos los objetos que se han aprendido hasta el momento: caramelos, vaso } \\
\text { con agua, zumo u otra bebida, pelota, un radiocasete y gusanitos. Se le pregunta a la alumna qué quiere, } \\
\text { esperando que haga uno de los signos. Cuando lo signe, se le entrega aquello que ha pedido. Se trata de } \\
\text { comprobar cuáles son los signos que ha asimilado hasta el momento sin necesidad de ayudas. }\end{array}$} \\
\hline Errores & \multicolumn{4}{|c|}{$\begin{array}{l}\text { - Errores en la forma de la mano, ya que no la abre del todo para designar el signo "gusanito". } \\
\text { - Errores de discriminación entre signos: sobre todo presenta errores de discriminación entre signos. } \\
\text { El signo "música" es el que menos ha asimilado, ya que ha necesitado moldeamiento para hacerlo } \\
\text { correctamente tanto en la posición como en la forma. }\end{array}$} \\
\hline \multirow{2}{*}{ Duración } & \multirow{2}{*}{30 minutos } & \multirow{2}{*}{ Ensayos } & En Bloque & 10 para gusanito \\
\hline & & & Aleatorios & 15 \\
\hline
\end{tabular}




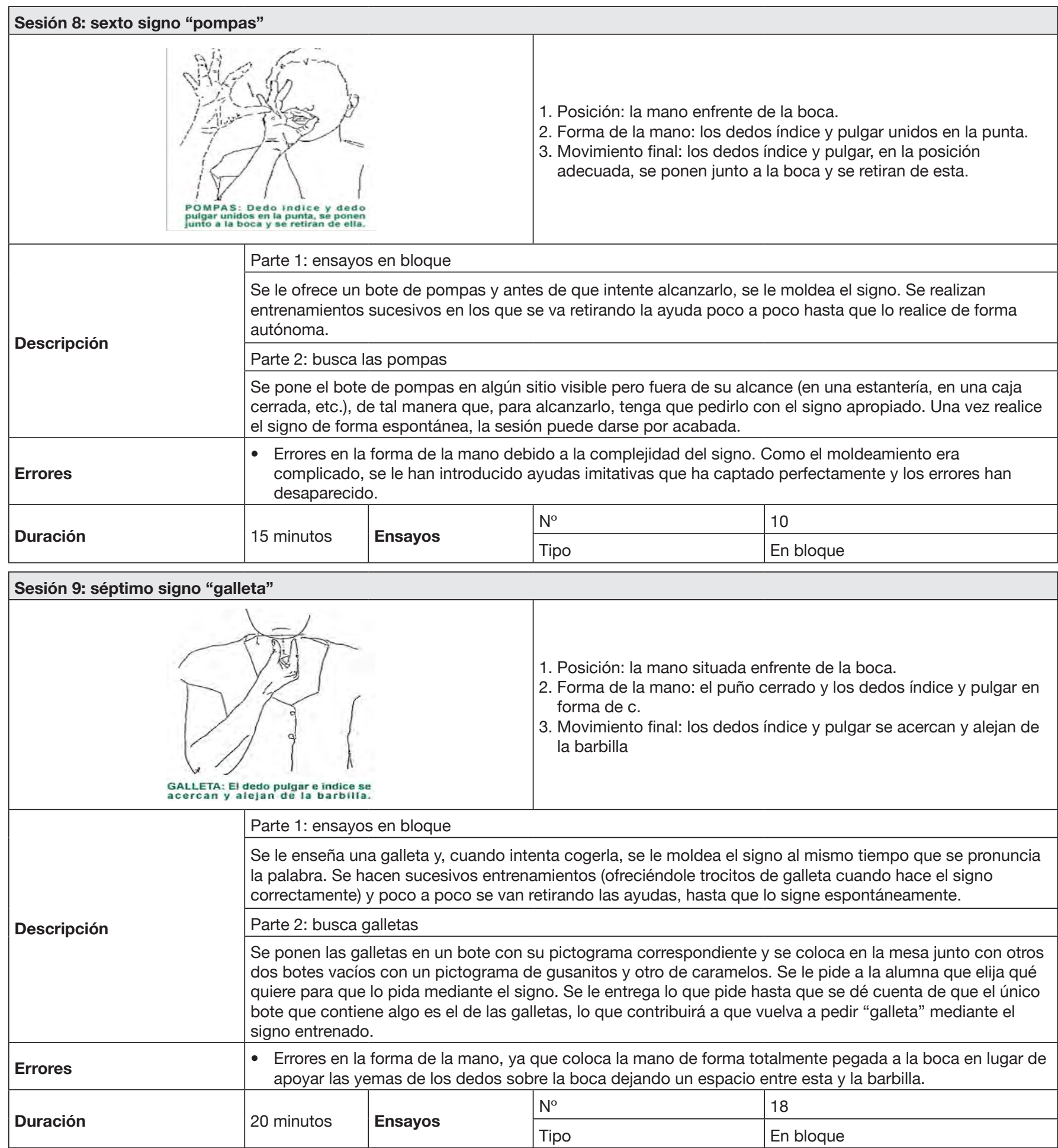




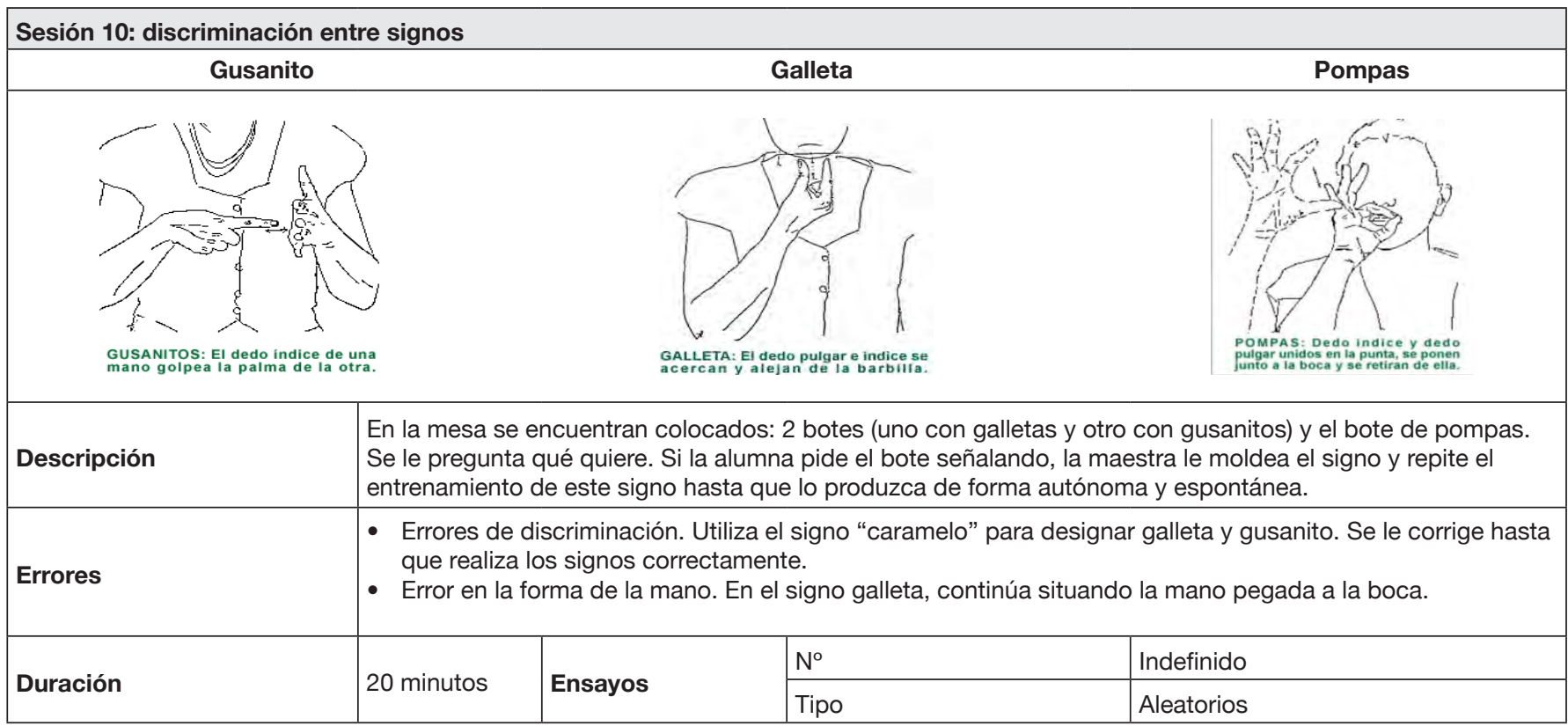

\begin{tabular}{|c|c|c|c|c|}
\hline \multicolumn{5}{|c|}{ Sesión 11: octavo signo "crema" } \\
\hline \multicolumn{3}{|c|}{$\begin{array}{l}\text { CREMA: Mano con la palma } \\
\text { hacia abajo, la otra la frota } \\
\text { como si echara crema. }\end{array}$} & \\
\hline \multirow{4}{*}{ Descripción } & \multicolumn{4}{|c|}{ Parte 1: ensayos en bloque } \\
\hline & \multicolumn{4}{|c|}{$\begin{array}{l}\text { Se le ofrece un tarro de crema y antes de que la alcance, se le moldean las manos en la posición y forma } \\
\text { adecuada, acompañado de la palabra crema. Se repite la acción y poco a poco se le retira la ayuda táctil. } \\
\text { Cada vez que realiza el signo de forma adecuada, se le pone un poco de crema en las manos y se le retira } \\
\text { para que la vuelva a pedir. }\end{array}$} \\
\hline & \multicolumn{4}{|c|}{ Parte 2:discriminación de signos } \\
\hline & \multicolumn{4}{|c|}{$\begin{array}{l}\text { Se colocan todos los objetos ya trabajados y se pregunta qué quiere. Posteriormente, se le van mostrando } \\
\text { objetos y se comprueba si realiza el signo apropiado. }\end{array}$} \\
\hline Errores & \multicolumn{4}{|c|}{$\begin{array}{l}\text { No ha realizado ningún error en el aprendizaje de crema. } \\
\text { - } \quad \text { Error de discriminación entre el signo galleta, música y caramelos por su similitud en la forma de la mano. }\end{array}$} \\
\hline \multirow[b]{2}{*}{ Duración } & \multirow[b]{2}{*}{25 minutos } & \multirow[b]{2}{*}{ Ensayos } & $\mathrm{N}^{\circ}$ & 5 para crema \\
\hline & & & Tipo & $\begin{array}{l}\text { En bloque y aleatorios en la } \\
\text { discriminación }\end{array}$ \\
\hline
\end{tabular}

Fuente: elaboración propia. 


\section{Sesión 12. La primera petición multisigno: "quiero+objeto"}

En la última sesión se ha introducido la primera petición multisigno: “quiero+objeto”. Se debe incluir cuando ya se dominen entre 8 y Io signos. Se expresan los mismos deseos, pero pero con una forma sintáctica más parecida a las expresiones en el lenguaje verbal (Ver Tabla 7).

\begin{tabular}{|c|c|c|c|c|}
\hline \multicolumn{5}{|c|}{ Sesión 12: la primera petición multisigno } \\
\hline \multirow{2}{*}{\multicolumn{2}{|c|}{$\begin{array}{l}\text { OUERER: Mano con la palma } \\
\text { hacia el pecho hacemos un } \\
\text { circulo a la lattura de este. }\end{array}$}} & \multicolumn{3}{|l|}{ Componentes } \\
\hline & & \multicolumn{3}{|c|}{$\begin{array}{l}\text { 1. Posición: la mano en el pecho. } \\
\text { 2. Forma de la mano: la mano con la palma de la mano abierta. } \\
\text { 3. Movimiento final: hacer un círculo a la altura del pecho. }\end{array}$} \\
\hline Descripción & \multicolumn{4}{|c|}{$\begin{array}{l}\text { En la mesa se colocan los objetos trabajados hasta el momento y se le pregunta qué quiere. Antes de que la } \\
\text { alumna realice el signo del objeto concreto, se le moldea el signo "quiero". Después se espera a que realice } \\
\text { el signo referente al objeto al mismo tiempo que el instructor pronuncia "quiero x (crema, pelota, beber...)" e } \\
\text { inmediatamente después se le entrega el objeto. Cuando la alumna consiga producir "quiero + objeto" con } \\
\text { regularidad y de forma espontánea, se puede hacer que pida otros objetos de la mesa siguiendo la misma técnica } \\
\text { de encadenamiento hacia atrás. }\end{array}$} \\
\hline Errores & \multicolumn{4}{|c|}{$\begin{array}{l}\text { - Errores de omisión: al principio de la sesión realiza los signos sin utilizar el "quiero" con un único signo. Sin } \\
\text { embargo, a medida que se incrementan los ensayos, se empiezan a utilizar los dos signos. }\end{array}$} \\
\hline \multirow{2}{*}{ Duración } & \multirow{2}{*}{20 minutos } & \multirow{2}{*}{ Ensayos } & $\mathrm{N}^{\circ}$ & 18 \\
\hline & & & Tipo & Aleatorios \\
\hline
\end{tabular}

Fuente: elaboración propia.

\section{Resultados}

- Evidenciar la presencia de conductas desafiantes con intención comunicativa

Antes de la intervención se registran un total de Io9 conductas problemáticas. El $70 \%$ son causadas por el déficit comunicativo de la alumna. En concreto, presenta conductas inadaptadas como consecuencia de no entender las instrucciones dadas por el adulto (comunicación receptiva: $22 \%$ ) y, sobre todo, por no disponer de un medio efectivo para poder expresar sus necesidades (comunicación productiva: $50 \%$ ). Estos resultados se muestran en la Figura I.

\section{Figura 1. Elementos del contexto (resultados de la observación sistemática pre-intervención)}

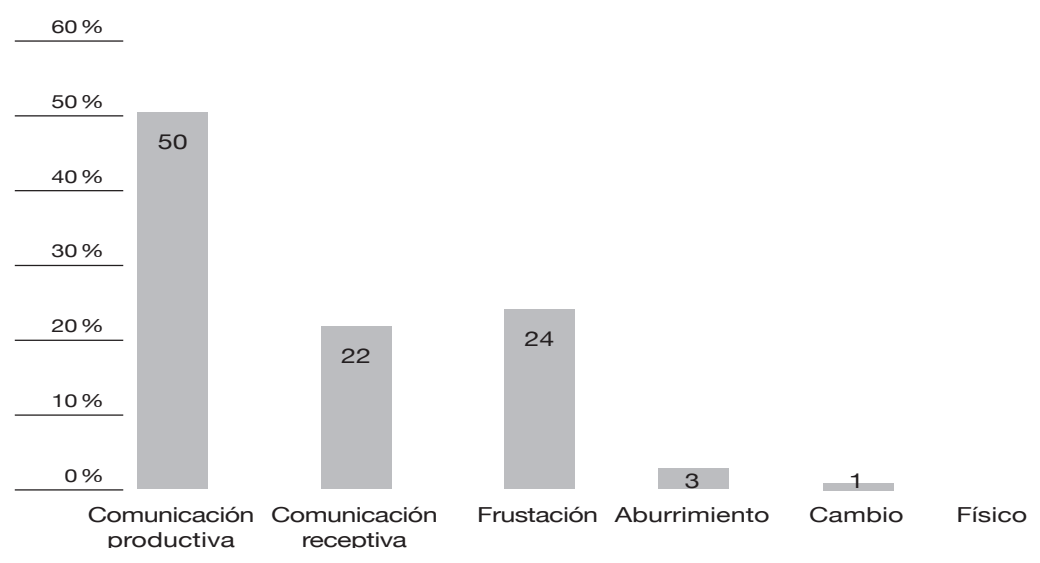

Fuente: elaboración propia. 
Por lo que respecta a las funciones de las conductas, la alumna las utiliza para obtener alguna cosa y, en menor medida, para evitar alguna tarea o llamar la atención, como se puede observar en la Figura 2.

\section{Figura 2. Funciones de las conductas problemáticas (resultados de la observación sistemática pre-intervención)}

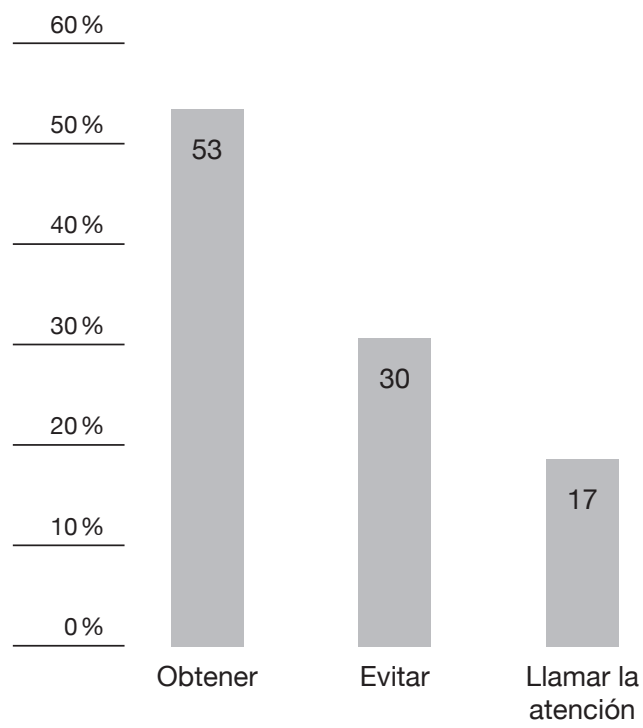

Fuente: elaboración propia.
Con estos resultados se evidencia la necesidad de que la alumna disponga de un medio eficaz que le permita expresar sus necesidades.

- Evaluación del programa de intervención: adquisición de los signos

Siguiendo los ítems de adquisición de signos, la alumna ha adquirido de forma completa cinco de los ocho signos entrenados: "beber", "pelota", "pompas", "galleta" y "crema" (Ver Tabla 8): los discrimina, hace un uso funcional de ellos para hacer peticiones y los ha generalizado en otros contextos del entorno escolar (zona de juego, de descanso, aula de música, patio, comedor, asamblea...), así como a otras personas (compañeros, maestras y educadora). Sin embargo, sigue confundiendo los signos: "caramelo", "música” y "gusanitos", debido a que la forma de la mano es la misma. A pesar de este error de discriminación, la alumna sí que ha conseguido hacer un uso funcional de los ocho signos entrenados, así como generalizarlos a otros contextos del centro escolar para pedir aquello que desea a distintas personas.

S también ha realizado avances como la pronunciación de la sílaba "be-be”, después

\begin{tabular}{|c|c|c|c|c|c|}
\hline \multirow[b]{2}{*}{ Signos } & \multirow[b]{2}{*}{ Discriminación } & \multirow[b]{2}{*}{ Uso funcional } & \multicolumn{2}{|c|}{ Generalización } & \multirow{2}{*}{$\begin{array}{l}\text { Adquisición } \\
\text { Total }\end{array}$} \\
\hline & & & $\begin{array}{c}\text { Otro } \\
\text { contexto }\end{array}$ & $\begin{array}{c}\text { Otra } \\
\text { persona }\end{array}$ & \\
\hline Caramelo & & $x$ & $x$ & $x$ & \\
\hline Beber & $x$ & $x$ & $x$ & $x$ & $x$ \\
\hline Pelota & $x$ & $x$ & $x$ & $x$ & $x$ \\
\hline Música & & $x$ & $x$ & $x$ & \\
\hline Pompas & $x$ & $x$ & $x$ & $x$ & $x$ \\
\hline Galleta & $x$ & $x$ & $x$ & $x$ & $x$ \\
\hline Gusanito & & $x$ & $x$ & $x$ & \\
\hline Crema & $x$ & $x$ & $x$ & $x$ & $x$ \\
\hline TOTAL & 5 & 8 & 8 & 8 & 5 \\
\hline
\end{tabular}

Fuente: elaboración propia. 
de varios ensayos del signo "beber", y de la sílaba "pa”, después de los del signo "pelota". La alumna ha progresado más rápido de lo esperado, en gran medida por su motivación hacia el programa, que lo asociaba a un momento lúdico y de diversión.

- Evaluación del programa de intervención: disminución de las conductas problemáticas

Hay una disminución de las conductas desafiantes (de Io9 en el registro pre, a 57 en el registro post) en todas los tipos de conducta, a excepción de agredir. Estos resultados se pueden observar en la Figura 3.

\section{Conclusiones y discusión}

En primer lugar, cabe destacar la utilidad que ha tenido la evaluación funcional de las conductas de la alumna, ya que a través de la observación directa y sistemática se ha establecido una relación entre la presencia de las conductas problemáticas y los elementos del contexto que propician su aparición (Gándara, 2007). En nuestro caso, se observa un déficit importante en la comunicación productiva y receptiva, y se constata la existencia de conductas problemáticas con una clara intención comunicativa (Matson et al., 2013).

Además, la evaluación de las conductas ha sido fundamental para elegir la intervención más adecuada y los reforzadores (actividades, objetos y signos motivadores) (Monfort, 2009; Valencia,

\section{Figura 3. Comparación de la frecuencia de aparición de conductas desafiantes antes y después de la} introducción del SAAC

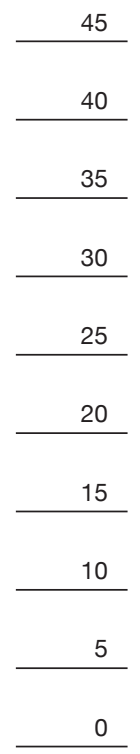

0

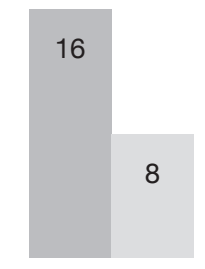

Autolesionarse

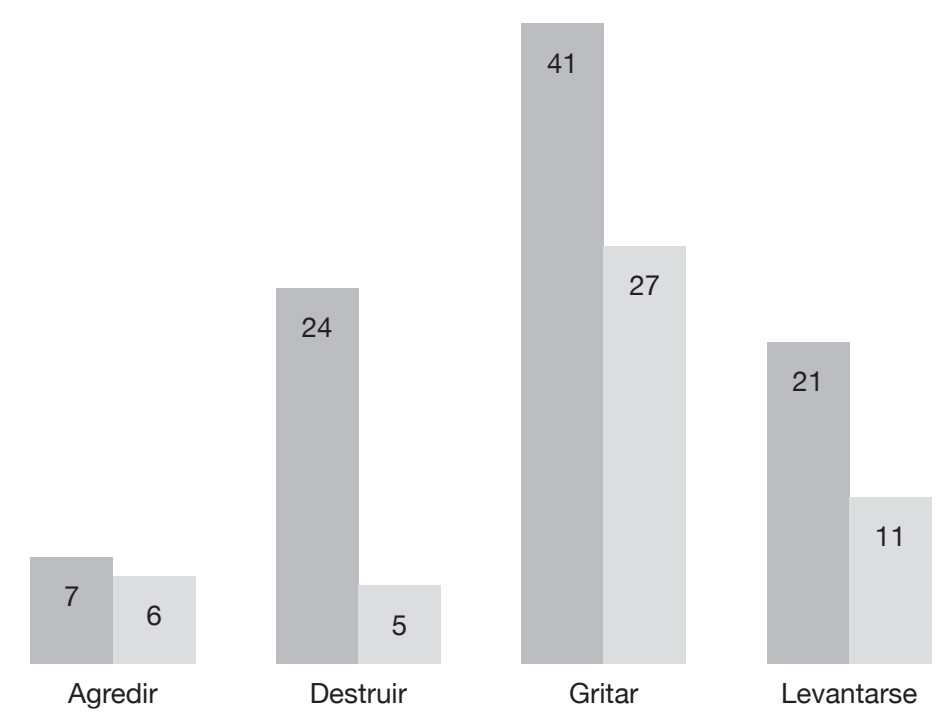

Pre-intervención

Post-intervención

Fuente: elaboración propia. 
20I4). La dotación del SAAC ha tenido resultados satisfactorios para el devenir diario de S (Regis y Callejón, 20I 5 ).

En el desarrollo del programa hemos observado las dificultades y los errores para aprender, discriminar y utilizar los distintos signos, así como las estrategias más efectivas, lo que es muy importante para mejorar y ajustar el programa, ya que la eficacia de un programa reside en la forma de instruirlo (Regis y Callejon, 201 5 ). Los resultados obtenidos son esperanzadores y creemos que mejorando el programa y cumplimentando sus tres fases, no sólo la primera, $S$ podría llegar a adquirir un lenguaje verbal funcional (García y Sánchez, 2003; Banoy, 2013; Fortea et al., 201 5 ).

Por lo que respecta a las técnicas de instrucción, las ayudas verbales han sido eficaces en la discriminación de signos y constituyen la esencia del programa, ya que la Comunicación Total consiste precisamente en eso: producir signos y palabras de forma simultánea (Monfort, 2006; Regis y Callejón, 20I5).

Organizar el contexto (rincón de comunicación) e introducir ayudas visuales ha sido esencial para el correcto desarrollo de las sesiones (Hodgdon, 2002). Asimismo, su motivación e interés propiciaron que, autónomamente y sin previa instrucción, pidiera las sesiones mediante un pictograma que se incluyó en el panel de peticiones del aula CyL. El programa se convirtió en un premio y en un instrumento para regular su conducta.
Con la comparación de los resultados obtenidos en la observación sistemática antes y después de la intervención se ha podido confirmar la hipótesis planteada inicialmente, ya que después de la dotación del SAAC se han reducido las conductas desafiantes (Emerson y Einfeld, 20I I). La alumna ha ido sustituyendo las conductas inadaptadas por la utilización de signos. Por tanto, el hecho de disponer de un SAAC con el que comunicar aquello que desea ha propiciado una reducción de las situaciones de frustración y angustia, incrementando la calidad de vida de la alumna.

Sin embargo, hay pocas investigaciones relacionadas con el tema de nuestra investigación. Por tanto, sería interesante continuar con investigaciones en el ámbito escolar que ampliaran la duración del programa y el número de participantes. Nuestra muestra es muy limitada, ya que únicamente es un estudio de caso y sus resultados no se pueden generalizar con una muestra tan reducida y por la gran variabilidad del alumnado con TEA (Monfort, 2009).

En definitiva, con esta investigación se evidencia la necesidad de que cualquier alumno con TEA disponga de un sistema de comunicación que le permita establecer relaciones con el medio que le rodea porque "hay duros silencios cargados que ocultan un rico mundo interior, hay también silencios estruendosos para los demás, lo que no hay son silencios impuestos que sean aceptables para los profesionales y los familiares que viven con personas con necesidades educativas especiales” (Rivière, I993). 
APA (2013): Guía de consulta de los criterios diagnósticos del DSM-5. Washington: Asociación de Psiquiatría Americana.

Banoy, L. (2013): Estrategias pedagógicas para la enseñanza de la comunicación alternativa y/o aumentativa en niños autistas (tesis digitales). Universidad Pedagógica Nacional, Bogotá, Colombia.

Barrios, S. (20I3): “Ayúdame a comprender el mundo. Apoyos visuales para la promoción de la autonomía en personas con trastornos del espectro del autismo y trastornos específicos del lenguaje”. TOG, Iо (8): 92-I04.

Calderón, L. et al. (2OI2): "Aportes desde la teoría de la mente y la función ejecutiva a la comprensión de los trastornos del espectro autista”. Revista CES Psicología, 5 (I): 77-99.

Carr, E. et al. (1996): Intervención comunicativa sobre los problemas de comportamiento. Madrid: Alianza.

Chiang, H. M. (2008): "Communicative spontaneity of children with autism". Autism, I 2 (I): 9-2I.

Emerson, E. y Enfield, S. (20I I): Challenging Behavior. New York: Cambridge University Press.

Escudero, A. et al. (2013): "Aparición y desarrollo de la atención conjunta en la infancia”. Anales de Psicología, 29 (2): 404-4I2.

Etchepareborda, M. C. (200I): "Perfiles neurocognitivos del espectro autista”. Revista de Neurología Clínica, 2: 175-192.

Ferrer, A. M. et al. (2010): "Intervención psicoeducativa en Trastorno de Espectro Autista”, en Grau, C. y Gil, D. (coords.): Intervención psicoeducativa en necesidades especificas de apoyo educativo. Madrid: Pearson.

Fortea, M. S. et al. (2OI 5): "Desarrollo temprano del lenguaje en niños pequeños con trastorno del espectro autista mediante el uso de sistemas alternativos”. Revista de neurología, 60 (Supl.I): 3I-35.

Gándara, C. (2007): "Principios y estrategias de intervención educativa en comunicación para personas con autismo: TEACCH". Revista de Logopedia, Foniatría y Audiología, 27 (4): 173I 86 .

Ganz, J. et al. (2OI 5): “Comparison between visual scene displays and exchange based communication in augmentative and alternative communication for children with ASD". Research in Autism Spectrum Disorders, I I: 27-4I.

Ganz, J. et al. (2OI2): “A meta-analysisof single case research studies on aided Augmentative and Alternative Communication Systems with individuals with Autism Spectrum Disorders". J Autism Dev Disord, 42: 60-74.

García, E. y Sánchez, M. M. (2003): "Programa de Comunicación Total y su incidencia en la aparición y desarrollo del lenguaje oral”, en I Jornadas de Comunicación Aumentativa y Alternativa. Consejería de Educación y Cultura, Servicio de Atención a la Diversidad de la Región de Murcia.

Garrido, D. et al. (201 5): "Dificultades de comprensión del lenguaje en niños no verbales con trastornos del espectro autista y sus implicaciones en la calidad de vida familiar". Revista de Neurología, 60 (5): 207-2I4.

González, M. et al. (20I4): "Sistemas de comunicación no verbales. Enriqueciendo los lenguajes Aumentativos y Alternativos con propiedades de accesibilidad y usabilidad". Revista informes científicos técnicos, 6 (2): 30-56.

Hodgdon, L. (2002): Estrategias visuales para mejorar la comunicación: Ayudas prácticas para la escuela y el hogar. EEUU: Quirk Roberts Publishing.

Ibáñez, A. (2005): “TEA, funciones ejecutivas y mentalismo: Reconsiderando la heurística de la descomposición modular". Revista Argentina de Neuropsicología, 6: 25-49. 
Jiménez, R. (2015): "Fundamentación psicológica sobre el desarrollo de habilidades sociales en escolares autistas desde la equinoterapia como medio de la actividad física adaptada”. Revista digital, 206.

López, J. et al. (20I4): "Prevalencia del trastorno negativista desafiante en España”. Revista de Psiquiatría y Salud Mental, 7 (2): 80-87.

López, S. y Rivas, R. (20I4): "El trastorno del espectro del autismo: Retos, oportunidades y necesidades". Informes psicológicos, I4 (2): I3-3 I.

Magiati, I. y Howlin, P. (2003): “A pilot evaluation study of the Picture Exchange Communication System (PECS) for children with autistic spectrum disorders”. Autism, 7 (3): 297-320.

Márquez, C. (2000): Documento para el debate. Parámetros de buena práctica del profesional del autismo ante las conductas desafiantes. Vigo: Actas del X Congreso de AETAPI.

Martos, J. y Llorente, M. (20I3): “Tratamiento de los trastornos del espectro autista: unión entre la comprensión y la práctica basada en la evidencia”. Revista de Neurología, 56 (Supl.r): 85-9I.

Martos, J. y Ayuda, R. (2002): “Comunicación y lenguaje en el espectro autista: el autismo y la disfasia”. Revista de Neurología, 34 (I): 58-63.

Matson, J. L. et al. (2013): "Moderating effects of challenging behaviors and communication deficits on social skills in children diagnosed with an autism spectrum disorder". Research in Autism Spectrum Disorders, 7 (I): 23-28.

Matson, J. L. et al. (2009): "The relation of communication and challenging behaviours in infants and toddlers with autism spectrum disorders". Journal of Development and Physical Disabilities, 2I: 253-26I.

Mesibov, G. y Howley, M. (20I I): El acceso al Currículo para alumnos con TEA. Programa TEACCH. Ávila: Autismo Ávila.

Monfort, M. (2009): “Comunicación y lenguaje: bidireccionalidad en la intervención en niños con trastorno de espectro autista”. Revista de Neurología, 48 (Supl. 2): 53-56.

Monfort, M. (2006): La práctica de la Comunicación Bimodal. Del signo a la palabra. Madrid: Editorial Entha.
Moreno, N. (2OI3): “Trastornos del lenguaje. Diagnóstico y tratamiento”. Revista de Neurología, 57 (I): 85-94.

Muñoz, J. A. (20I3): “Comprender, prevenir y afrontar las conductas desafiantes en niños con trastornos del espectro autista”, en Alcantud, F. (coord.): Trastornos del espectro autista: detección, diagnóstico e intervención temprana. Madrid: Pirámide.

O'Reilly, M. et al. (20I2): "Examination of an antecedent communication intervention to reduce tangibly maintained challenging behaviour: a controlled analog analysis". Research in Developmental Disabilities, 33: I $462-$ I 468

Regis, P. J. y Callejón, M. D. (20I 5 ): “Del pictograma a la imagen: herramientas de comunicación y lenguaje en personas con síndrome de Asperger a través de recursos visuales para la inclusión social". ArteterapiaPapeles de arteterapia y educación artística para la inclusión social, Iо: 329-34I.

Rivèire, A. (1993): "Prólogo", en Sotillo, M. (coord.): Sistemas alternativos de comunicación. Madrid: Trotta.

Schaeffer, B. et al. (2005): Habla signada para alumnos no verbales. Madrid: Alianza.

Soto, R. (2007): “Comunicación y lenguaje en personas que se ubican dentro del espectro autista". Revista Electrónica "Actualidades Investigativas en Educación”, 7 (2): I-I6.

Valencia, S. E. (20I4): Los sistemas aumentativos y alternativos y su relación con la comunicación de los jóvenes con discapacidad intelectual de I5 a 20 años que asisten a la fundación "calidad de vida independiente" (tesis doctoral). Universidad Nacional de Loja, Ecuador.

Verpoorten, R. et al. (20I4): ComFor. Precursores de la comunicación. Prueba para la evaluación de las necesidades de sistemas aumentativos de comunicación. Ávila: Autismo Ávila.

Villanueva, C. et al. (2016): "Efectos de un programa piloto de desarrollo cognitivo 'teoría de la mente' en tres niños con autismo: componente emocional”. Revista de neurología, 62 (2): $267-272$ 